\title{
Children's Smart Clothing Design Pattern and Development
}

\author{
Ping Wang \\ Department of Art, Guangdong University of Science \& Technology, Dongguan, China \\ 28993716@qq.com
}

Keywords: artificial intelligence; children's clothing; design

\begin{abstract}
From the prevalence of VR technology in 2016 to the fire of artificial intelligence in 2017, the development track of smart high-tech has always shown a rapid expansion. In 2018, the Prime Minister responded to reporters during the two sessions, focusing on the concept of "smart +". All kinds of signs show that the "smart" role in promoting national economic development and social and cultural prosperity cannot be underestimated. At present, the issue of child safety is a problem that needs to be paid attention to by millions of households in today's society. Through modern technology, garments have functions such as location tracking, which provides a good solution to the problem of children's safety in near-field loss. In this paper, based on the multi-dimensional consumer demand for children's clothes, combined with the performance of smart components, the combination of smart wearable devices and children's safety apparel is discussed, and a design flow architecture that takes into account both functional and aesthetic appeal is proposed. Through the analysis of the connection technology between smart clothing and mobile terminals, the idea of single interaction and multi-device sharing is proposed, and a multi-interactive intelligent wearable device development flow based on energy optimized configuration and high-efficiency information transmission is established.
\end{abstract}

\section{Introduction}

At the 4th China National Garment Technology Development Conference held recently, the children's smart clothing design won the award. Children's smart clothing can use data interactive communication sensing technology, adopt digital product and information service signal exchange and interactive feedback method, combine NFC, iBeacon, GPS, Bluetooth, modified fiber, luminous fiber and other new technologies, new technologies, intelligent security Elemental and popular trend predictions are integrated into the design of children's wear products based on basic practicality. The design has special safety features and is in line with the popular innovative children's smart clothing.

\section{Children's Smart Apparel Design and Research Status}

There are 367 million children in China and it is the world's "children's clothing power." The earliest research on smart devices can be traced back to the concept of smart vests introduced in the Computer Wearable Systems Research Center of the Massachusetts Institute of Technology's body 
laboratory in 1993, becoming the earliest intelligent smart device. At the end of the 20th century, a coat with headphones, mobile phones and MP3 came out. In the 1960s, the United States developed a "combat cotton" space space suit in the "Apo Year Plan" that can sense the sensory changes of the human body and the surrounding environment. In addition, the United States Sensatex has developed a smart thermal shirt that can monitor heart rate, body temperature, breathing, and how many calories are consumed. Tuisan Reima reports that Richard Palmer designed Mingfa smart automatic waterproof lifesaving children's smart clothing, which means that children have a strong security protection.

Looking at the domestic, Li Qiang of Tianjin Polytechnic University studied the model of human body temperature measurement based on light-based Bragg light, and concluded that the measured temperature in the smart clothing can be used as the lower temperature of clinical medicine. Donghua University Wu Xuekui pointed out that Jianli faces intelligence

The multi-physiological information fusion sentiment judgment model framework, discussed and realized the most intelligent multi-physiological information fusion emotion discrimination software platform to provide a harmonious human-computer interaction environment. Followed by the ever-evolving teachings and patents related to intelligent design research ${ }^{[1]}$.

Compared with the smart clothing research mentioned above, the field of smart element sand and children's clothing safety has lagged behind. The first research on children's intelligent safety clothing was to attach electronic sensing devices (components) to clothing. Since Professor Wen Liuyuan of Jiangnan University reported on the digital interactive design in smart clothing for school-aged children, it has been used as a new type of child intelligence. Under the interactive design of digital information established under the 1T2F model, garments have reduced the potential for child safety hazards caused by the external environment. In recent years, more and more studies have been conducted on the design of packaging designs ${ }^{[2]}$. This article starts from the modern design model $-1 \mathrm{~T} 2 \mathrm{~F}$, focuses on the intelligent design of the front loading and the new structural design, and reduces the external environment to the inconvenience and injury caused by the development of the child care through the new type of safety smart clothing.

\section{1T2F Fashion Design Concept}

The 1T2F model refers to the integration of traditional, fashion and functional clothing design concepts. To guide the design in 1T2F mode can not only deeply understand the traditional culture, but also make up for and promote the tradition through the clear definition of the concept;

Under the premise of aesthetics and current fashion trends, fashion will continue to be the core of the design concept, in order to activate the design atmosphere. Traditional clothing design focuses on basic practical functions, while the $1 \mathrm{~T} 2 \mathrm{~F}$ model abstracts abstract practical functions as figurative special safety functions, including not only basic practicality, but also the rational efficiency of intelligent science. This is the key point of the entire design concept. Using the combination of three elements, using tradition as a foundation, fashion as the core, and function as the key, making it a flaw in the original traditional design concept.

In recognition of the inevitability of the concept of modern mode, we can improve the modern children's wear design capabilities to design children's apparel products that combine both traditional taste and fashion while combining the safety benefits of intelligent science and enhance the added value of products.

\section{Children's Smart Clothing Design Pattern}

\subsection{Design Principles}

Based on the 1T2F model, the new smart and safe children's apparel design system should be based 
on children's characteristics. It should be based on the "children's wear" safety-oriented principle. It must be based on the design principles of wearing comfort, fashion and functional diversity, and fully consider the children's psychophysiology, human mechanics, Equipment flexibility, environmental factors, safety factors, as well as the function and structure of the apparel itself are designed in a human-oriented manner to minimize the burden on parents to supervise children. While saving time and costs, you can quickly react when your child is at risk or has a strange body. In the prevention of accidents, it can also provide a basis for the later diagnosis of the disease.

\subsection{Design Ideas}

The specific process design is as follows: First, smart clothing design must be integrated and designed for the monitoring target, and the monitoring program should be selected.

Monitoring parameters thus design functional modules. Then combine each function module with the loading body, according to the selected sensor design and placement of the signal and signal transmission methods, in order to obtain a good and stable data acquisition, and finally complete the uniform design.

\subsection{Process Design}

The design consists of a garment body, a temperature sensor device and a humidity sensor. The line is concealed in the garment and is carried by a flat wire without causing discomfort ${ }^{[3]}$. The probe is in the form of a patch, which is different from the icy feeling of the thermometer that can cause children's discomfort. This kind of probe can contact the skin for a long time, more humanized than the same type of design, better clothing performance. The process of a specific functional module and garment prototype assembly is shown in Figure 1 below. The whole installation design, the cartoon pattern is loaded into the host in the form of a hollow pocket, there is a small hole for the wire to pass through, arrive at the monitoring site, the temperature sensor is placed under, and fixed by hand-sewing; the body temperature sensor amplification circuit has been integrated in the sensor, so the acquisition The incoming signal is transmitted directly to the junction box, which is integrated and transmitted to the monitoring center via a data acquisition card. This system uses wired transmission to connect the junction box and the computer protection center.
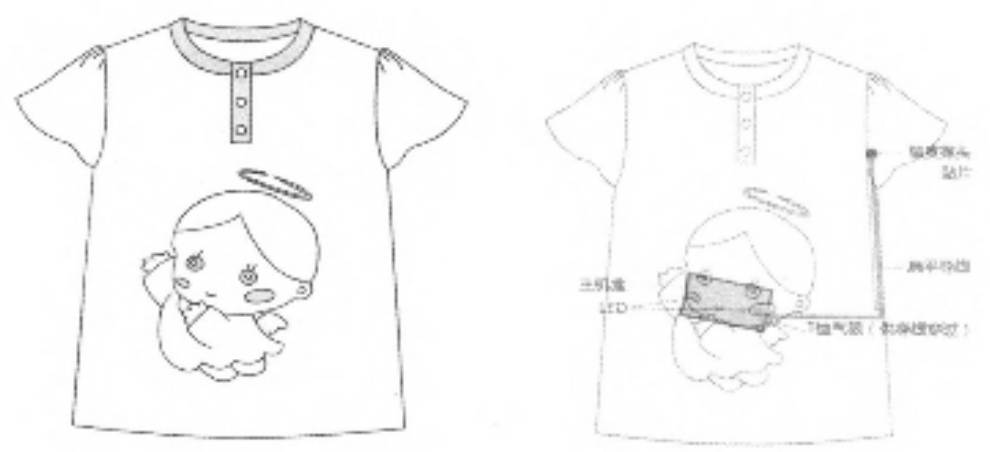

Figure 1 The approximate position of the functional module on the garment surface 


\section{Children's Smart Clothing Design and Development}

\subsection{Children's smart clothing to prevent children from falling}

Children are characterized by liveliness and weak self-protection. They often cause damage due to chasing, running, jumping and other outdoor activities. The knee is a site where children's sports are easily hurt. In today's children's clothing market, children's clothing styles are mostly taken into consideration. Children's clothing fabrics or children's local vulnerable areas (such as knees, ankles, elbows, etc.) are usually not subjected to special treatment. In order to overcome the shortcomings of existing children's clothing, based on the $112 \mathrm{~F}$ design concept of clothing to provide a personalized protective children's trousers to prevent injuries to the body parts, which has a higher comfort and aesthetics.

\subsection{Children's smart clothing to monitor physical changes}

The past clothing system functions are simple and unwieldy. The improved intelligent monitoring clothing can realize the remote transmission of multiple data, mainly for infants and young children. The design can be specifically designed for children's physical changes smart clothing design ${ }^{[4]}$. The garment is a cartoon pattern similar to a pocket. The light emitting diode is set in the face of the cartoon pattern. Its status is constantly on, blinking and flashing.

Three kinds of sparkling, controlled by the sensor, its state depends on the temperature detector's perception of children's body surface temperature. The default setting program is that the 1ED lamp lights steadily at $38^{\circ} \mathrm{C}$, blinks at $39^{\circ} \mathrm{C}$, flashes rapidly at $40^{\circ} \mathrm{C}$ and sounds an alarm. When the set temperature is reached, the red light of the pattern flashes, and the cartoon pattern has a flush face, which vividly represents the current situation. The guardian holds the terminal in real time, displays the detected temperature in real time, and can set the alarm temperature freely. Once the set value is reached, it will prompt and monitor for a long time; the humidity sensor is mainly used to remind the guardian to change the underpants or diapers to the child in time to avoid the wetness. Without timely care, it can cause colds, skin eczema, etc. The chip transmits urinary wetness information to the receiver and sends out music, which not only serves as a reminder but also helps the baby's intellectual development.

\section{3 children's smart clothing that can change sleeves}

Most of the existing children's clothing is connected to the sleeves of the clothing body, and even if it is detachable, it is mostly a decorative function of the clothing. The child's self-control and self-care ability is poor. Often, only part of the children's clothing needs to be replaced, or the writing and drawing postures are incorrect, causing the clothing to be partially unclean, and the entire clothing is often required to be frequently cleaned. Due to various factors, such as the weather (such as winter and rainy weather) and school rules and regulations (the school uniform must be worn from Monday to Friday), the existing children's clothing can hardly meet the needs of children and parents. In order to overcome the shortcomings of the existing children's clothing, a personalized detachable sleeve type children's clothing is provided, and a replaceable sleeve is designed for the vulnerable parts (sleeves) of children's clothing. Figure 2 below shows children's smart clothing that can replace the sleeves. 


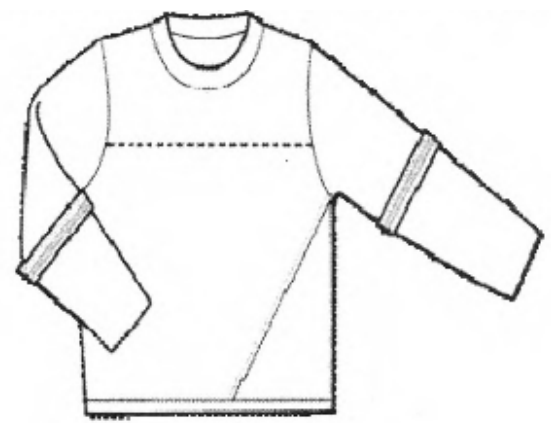

Figure 2 Children's smart clothing with changeable sleeves

\section{Conclusion}

Based on the new model of the new children's intelligent safety apparel design under the $112 \mathrm{~F}$ model, it is proposed that the new heavy apparel design should follow the design principles and standards of intelligence, comfort, safety, and systemic design. The smart and safe children's clothing design is a once in the history of children's wear design ${ }^{[5]}$. The major innovations promoted by the concept of smart security have become more deeply rooted in the entire textile and apparel industry. The perfect combination of smart materials, electronic information equipment and children's clothing is more and more intelligent clothing itself, more and more stylish appearance, more and more humanized design, giving children more features of clothing, but also greatly facilitates the guardian's Life and work.

\section{References}

[1] Zhang Xiying, Tong Xiaqing. Safety symbols in children's clothing [C]. China Knitting Industry Association, 2014: 345-351.

[2] Fang Donggen. Application and R\&D Concept of Sensor Device in Children's Safety Clothing[C]. China Knitting Industry Association, 2014:351-358.

[3] Tu Cheng. Discussion on children's clothing quality safety and standardization system [C]. China Standards Association, 2013:458-461.

[4] Shen Lei, Hong Wenjin. Application of Intelligent Fiber in the Design of Smart Garment Design[C]. China Textile Engineering Society, Jiangsu Textile Engineering Society, 2014:51-55.

[5] Yang Qi, Zou Lili, He Zhiyong. Research on the Comparison of Children's Apparel Domestic and Foreign Advanced Standards [C]. China Standards Association, 2015. 